\title{
Therapie mit Dexamethason bei Patienten mit COVID-19
}

\author{
Positionspapier der Deutschen Gesellschaft für Pneumologie und \\ Beatmungsmedizin e. V.

\section{Treatment with Dexamethasone in Patients with COVID-19}

\author{
A Position Paper of the German Respiratory Society (DGP)
}

Autoren

M. Kolditz ${ }^{1}$, D. Dellweg ${ }^{2}$, H. Geerdes-Fenge ${ }^{3}$, P. M. Lepper ${ }^{4}$, T. Schaberg ${ }^{5}$, S. Ewig ${ }^{6}$, M. Pfeifer ${ }^{7,8,9}$, T. Bauer $^{10}$

Institute

1 Universitätsklinikum Carl Gustav Carus an der Technischen Universität Dresden, Klinik und Poliklinik für Innere Medizin I, Dresden

2 Fachkrankenhaus Kloster Grafschaft GmbH, Akademisches Lehrkrankenhaus der Philipps-Universität Marburg, Schmallenberg Grafschaft

3 Universitätsmedizin Rostock, Zentrum für Innere Medizin, Abteilung für Infektionskrankheiten und Tropenmedizin, Rostock

4 Universitätsklinikum des Saarlandes, Innere Medizin V Pneumologie, Allergologie, Beatmungs- und Umweltmedizin, Homburg/Saar

5 Rotenburg (Wümme)

6 Thoraxzentrum Ruhrgebiet, Department of Respiratory and Infectious Diseases, EVK Herne and AugustaKrankenanstalt Bochum, Bochum

7 Klinik und Poliklinik für Innere Medizin II, Universitätsklinik Regensburg, Regensburg

8 Abteilung für Pneumologie, Fachklinik für Lungenerkrankungen Donaustauf
9 Krankenhaus Barmherzige Brüder, Klinik für Pneumologie und konservative Intensivmedizin, Regensburg

10 Helios Klinikum Emil von Behring GmbH, Lungenklinik Heckeshorn, Berlin

Bibliografie

DOI https://doi.org/10.1055/a-1216-5739 |

Online-Publikation: 16.7.2020 |

Pneumologie 2020; 74: 493-495

(C) Georg Thieme Verlag KG Stuttgart · New York ISSN 0934-8387

Korrespondenzadresse

PD Dr. med. Martin Kolditz, Universitätsklinikum

Carl Gustav Carus, Technische Universität Dresden,

Klinik und Poliklinik für Innere Medizin I,

Fetscherstr. 74, 01307 Dresden

martin.kolditz@uniklinikum-dresden.de
HINWEIS

Eine aktualisierte Version dieses Beitrags finden Sie unter: https://pneumologie.de/covid-19.

\section{Hintergrund}

Der Erkrankungsverlauf einer Infektion mit dem neuartigen Coronavirus SARS-CoV-2 (COVID-19) kann in verschiedene Phasen unterteilt werden. Die frühe Form die Erkrankung wird durch die Virusinfektion und -replikation dominiert. Bei einem Teil der Patienten können im späteren Krankheitsstadium ausgeprägte proinflammatorische Prozesse getriggert werden, welche mit zunehmendem pulmonalem und teils auch extrapulmonalem Organversagen assoziiert sind [1,2]. Bei diesen Patienten können antiinflammatorische Therapiestrategien erfolgversprechend sein und werden aktuell in verschiedenen Studien getestet.
Bisher bestanden Bedenken gegenüber einem Einsatz von Steroiden bei COVID-19 außerhalb etablierter klinischer Indikationen wie einer zusätzlich exazerbierten obstruktiven Atemwegserkrankung oder dem therapierefraktären septischen Schock. Diese beruhten überwiegend auf Daten von Beobachtungsstudien, welche - meist bei Infektionen mit anderen Viren - eine Assoziation mit verzögerter Viruselimination oder bakteriellen Superinfektionen beschrieben, ohne dass die Letalität signifikant beeinflusst wurde [3,4].

Nun berichtet eine große britische randomisierte Studie von einem Überlebensvorteil hospitalisierter Patienten mit COVID19 unter Dexamethason [5]. Somit wird eine Neubewertung erforderlich.

\section{Recovery-Studie}

Die Recovery-Studie ist eine mehrarmige, adaptierbare, offene, multizentrische (176 Zentren), randomisierte Studie, die verschiedene medikamentöse Interventionen im Vergleich zur 
Standardtherapie bei hospitalisierten Patienten mit vermuteter oder gesicherter COVID-19-Erkrankung evaluiert hat. Am 22.6. 2020 erfolgte auf einem Preprint-Server die bisher noch nicht begutachtete Publikation der vorläufigen Auswertung von 2104 Patienten, welche randomisiert eine Therapie mit $6 \mathrm{mg}$ Dexamethason pro Tag für bis zu 10 Tage zusätzlich zur Standardtherapie erhielten, im Vergleich zu 4321 Patienten, welche nur eine Standardtherapie erhielten (u.a. 24\% Azithromycin, kein Remdesivir). Ausgeschlossen von der Randomisierung in den Dexamethason-Arm der mehrarmigen Gesamtstudie mit insgesamt 11320 Patienten wurden Patienten mit einer definitiven Indikation für oder mit einer Kontraindikation gegen eine Therapie mit Dexamethason oder wenn Dexamethason lokal nicht verfügbar war (zusammen 17\%). Der primäre Endpunkt war die 28-Tages-Gesamtletalität. Die eingeschlossenen Patienten waren im Mittel 66 Jahre alt, $64 \%$ waren männlich, $21 \%$ hatten eine pulmonale Komorbidität. Bei Randomisierung erhielten $24 \%$ keine respiratorische Unterstützung, $60 \%$ eine Sauerstofftherapie und $16 \%$ eine mechanische Beatmung. Bei $82 \%$ war die Infektion mit SARS-CoV-2 virologisch nachgewiesen. Daten zu Biomarkern liegen nicht vor.

Die Studie referiert folgende Ergebnisse:

- Eine relative Reduktion der 28-Tages-Letalität in der Gesamtkohorte um $17 \%$ von $24,6 \%$ auf $21,6 \%$;

RR $0,83(0,74-0,92), p<0,001$

- Eine relative Reduktion der 28-Tages-Letalität bei initial beatmeten Patienten um 35 \% von 40,7 \% auf 29,0 \%; RR 0,65 (0,51-0,82), $\mathrm{p}<0,001$

- Eine relative Reduktion der 28-Tages-Letalität bei Patienten mit initialer Sauerstofftherapie um $20 \%$ von $25,0 \%$ auf $21,5 \%$; RR 0,80 (0,70-0,92), $p=0,002$

- Einen relativen Anstieg der 28-Tages-Letalität bei Patienten ohne initiale Sauerstofftherapie um $22 \%$ von $13,2 \%$ auf $17 \%$, RR 1,22 (0,93-1,61), $p=0,14$

\section{Vorläufige Bewertung der Studie}

Es handelt sich um eine sehr große, randomisierte, aber nicht verblindete Studie mit dem Endpunkt der Letalität.

Pathophysiologisch sind der nachgewiesene Effekt einer antiinflammatorischen Therapie bei fortgeschrittenem Krankheitsstadium (signifikanter Effekt nur bei - schlecht definierter - respiratorischer Insuffizienz sowie in der Subgruppe mit einer Symptomatik > 7 Tage zum Zeitpunkt der Randomisierung) und ein potenziell ungünstiger Effekt im früheren Erkrankungsstadium plausibel.

Die vorliegende Studiendokumentation ist als vorläufig anzusehen (nach Bericht Randomisierung bis 8.6.2020, Datenbankschluss bereits 10.6. 2020, dennoch Angabe von 95\% Vollständigkeit der Verlaufsdokumentation bezüglich des Endpunktes), die aktuelle Bewertung bedarf daher einer Re-Evaluation nach Vorliegen aller Daten.

Das Studiendesign war auf eine unkomplizierte und rasche Randomisierung möglichst vieler Patienten in einer Situation eines durch die Pandemie stark belasteten Gesundheitssystems ausgelegt, dafür wurden erhebliche Einschränkungen bezüglich der detaillierten Erfassung der klinischen Charakteristika der eingeschlossenen Patienten, des klinischen Verlaufs der Erkrankung und der Beschreibung der Standardtherapie in Kauf genommen, die die Dateninterpretation limitieren. Soweit den verfügbaren Daten zu entnehmen ist, existierten auch keinerlei standardisierte Vorgaben zum Management der respiratorischen Insuffizienz innerhalb der Studienzentren. Dies ist kritisch insbesondere vor dem Hintergrund, dass die Einteilung der respiratorischen Insuffizienz (kein Sauerstoff, Sauerstoff, mechanische Beatmung) nach ihrer Therapie und nicht nach ihrem objektivierbaren Schweregrad erfolgte.

Der Verlauf von COVID-19 ist variabel, sodass damit gerechnet werden muss, dass ein Patient, der zum Zeitpunkt der Randomisierung keinen Sauerstoff erhielt, im späteren Verlauf Sauerstoff benötigte und/oder maschinell beatmet werden musste. In dem aktuell vorliegenden Manuskript finden sich jedoch keine klaren Angaben bezüglich einer Steroidgabe bei einem Stadienwechsel.

Weitere Kritikpunkte der Studie umfassen den unverblindeten Medikamenteneinsatz, die bisher fehlende Auswertung von Subgruppen (z.B. mit bzw. ohne pulmonale Komorbidität), die fehlenden Daten zu (z. B. inflammatorischen) Biomarkern sowie die auf Deutschland nicht direkt übertragbaren Letalitätsraten insbesondere in der Standardtherapiegruppe bei Patienten ohne respiratorische Insuffizienz. 17\% der potentiell randomisierbaren Patienten wurden ausgeschlossen, weil eine Indikation oder Kontraindikation für Dexamethason vorlag oder Dexamethason nicht verfügbar war, ohne dass dies im Bericht spezifiziert dargestellt wird. $9 \%$ der Patienten hatten einen negativen SARS-CoV-2-Test, bei weiteren $9 \%$ gab es zum Zeitpunkt der Studie noch kein Ergebnis; leider wurden diese Patienten nicht näher charakterisiert. Daten zu Nebenwirkungen fehlen vollständig, und eine detaillierte Aufarbeitung ist nach Studienprotokoll auch nicht zu erwarten. Auch Daten zum längeren Follow-up noch hospitalisierter Patienten sind notwendig.

Diese Einschränkungen sind nach Ansicht der Autorengruppe als relevante Mängel der Studie zu bewerten, die allerdings im Kontext der im Studiendesign festgelegten Priorisierung eines raschen Einschlusses mehrerer tausend Patienten innerhalb weniger Wochen bei dringlich notwendiger Evidenzgenerierung abgewogen werden müssen. Die hohe Anzahl der randomisierten Patienten und der Letalitätsendpunkt lassen es dennoch aus Sicht der Autorengruppe als geboten erscheinen, Konsequenzen für die Patientenversorgung auf der Basis dieser vorläufigen Bewertung abzuleiten.

\section{Einordnung der Studie}

Die Dosis von $6 \mathrm{mg}$ Dexamethason/Tag entspricht in etwa $180 \mathrm{mg}$ Hydrokortison, was aus Sicht potenzieller Nebenwirkungen weniger problematisch erscheint und in etwa der empfohlenen Therapie beim therapierefraktären septischen Schock entspricht.

Die bisherige Studienlage außerhalb der etablierten Indikation eines refraktären septischen Schocks für Patienten mit ambulant erworbener Pneumonie ist uneinheitlich und lässt keine klare Empfehlung zu. Für Patienten mit ARDS war die Verwen- 
dung von Steroiden gemäß einer Cochrane Meta-Analyse ( $n=7$ Studien mit insgesamt 851 Patienten) [6] mit einer Reduktion der Letalität vergesellschaftet (RR 0,$75 ; 95 \% \mathrm{Cl} 0,59-0,95)$. Eine kürzlich veröffentlichte prospektiv-randomisierte Studie an 277 Patienten mit ARDS und überwiegend zugrundeliegender bakterieller Infektion zeigte ebenfalls eine signifikante Letalitätsreduktion unter Dexamethason im Vergleich zu Placebo [7]. Hier waren die Patienten im Dexamethason-Arm mit $20 \mathrm{mg}$ über 5 Tage und mit $10 \mathrm{mg}$ über weitere 5 Tage behandelt worden. Der Effekt auf die Letalität war in der Gruppe der beatmeten Patienten signifikant. In weiteren Studien zum ARDS mit unterschiedlichen Interventionen [8-10] erhielten die Patienten in allen Studienarmen Steroide zwischen $16 \%$ im Jahr 2010 [8] und 67,5\% im Jahr 2018 [10]. Die Gabe von Steroiden bei Patienten mit schwerem ARDS entspricht somit teils gängiger Praxis. Die Studien fokussierten jedoch überwiegend auf nicht viral bedingtes ARDS, und eine Übertragbarkeit auf ein ARDS bei COVID-19 kann nicht vorausgesetzt werden.

Vor diesem Hintergrund muss der Anteil von Patienten mit Steroidgabe im Standardtherapiearm der Recovery-Studie von $7 \%$ betrachtet werden.

\section{Empfehlung der Autorengruppe zum Einsatz von Dexamethason bei COVID-19}

Auf der Basis der vorläufigen Bewertung dieser noch nicht begutachteten und nicht finalen Studienvorveröffentlichung:

1. empfehlen wir keinen Einsatz von Dexamethason bei ambulanten Patienten oder bei hospitalisierten Patienten mit COVID-19 ohne eine Indikation für eine Sauerstoffgabe und ohne andere klinische Indikation, da hier eine Übersterblichkeit nicht ausgeschlossen werden kann und die Effekte einer reduzierten Viruselimination potenziell überwiegen.

2. empfehlen wir in Analogie zu den vorläufigen Daten der Recovery-Studie den Einsatz von Dexamethason $6 \mathrm{mg} / \mathrm{d}$ p. o. oder i.v. für bis zu 10 Tage bei Patienten mit COVID-19 und manifester schwerer respiratorischer Insuffizienz mit der Indikation zur Sauerstoffgabe oder zur Beatmung, sofern keine Kontraindikationen vorliegen. Zu beachten ist hierunter die Notwendigkeit einer engmaschigen Kontrolle und Einstellung der Blutglukose und des Serumnatriums, einer regelmäßigen Evaluation potenzieller Superinfektionen sowie das erhöhte gastrointestinale Blutungsrisiko [11,12].

3. halten wir zum jetzigen Zeitpunkt eine Empfehlung für oder gegen eine Dexamethasongabe bei COVID-19-Patienten mit nicht klar definierter respiratorischer Insuffizienz (in dieser Studie $\mathrm{SpO}_{2}$ unter 92 - $94 \%$ ) für nicht gerechtfertigt. Hier sollte die Entscheidung, eine Steroidtherapie zu initiieren, individuell auf einer mehrdimensionalen Risiko-Nutzen-Abwägung hinsichtlich der respiratorischen Insuffizienz, basierend auf z. B. Atemfrequenz, Ausmaß der CT-Verschattungen, bereits etablierte prognostische Parameter, kurzfristiger Verlauf und Komorbiditäten, getroffen werden.

4. Nicht berührt hiervon ist die unabhängig bestehende Indikation zur Steroidtherapie bei zusätzlicher exazerbierter obstruktiver Atemwegserkrankung und beim therapierefraktären septischen Schock.

Die vorliegende Stellungnahme gibt den Stand der Daten zum Zeitpunkt Ihrer ersten Veröffentlichung am 1.7.2020 wieder. Eine Re-Evaluation dieser Empfehlung soll auf der Basis der begutachteten Vollveröffentlichung der Daten erfolgen.

Interessenkonflikt

Die Autorinnen/Autoren geben an, dass kein Interessenkonflikt besteht.

Literatur

[1] Fachgruppe COVRIIN am RKI. Wann könnte die Einleitung einer antiviralen Therapie gerechtfertigt werden? Stand: 24.06.2020. doi: $10.25646 / 6962$

[2] Messner CB, Demichev V, Wendisch D et al. Ultra-high-throughput clinical proteomics reveals classifiers of COVID-19 infection. Cell Systems 2020. doi:10.1016/j.cels.2020.05.012

[3] Li H, Chen C, Hu F et al. Impact of corticosteroid therapy on outcomes of persons with SARS-CoV-2, SARS-CoV, or MERS-CoV infection: a systematic review and meta-analysis. Leukemia 2020; 34: 15031511. https://doi.org/10.1038/s41375-020-0848-3

[4] Giacobbe DR, Battaglini D, Ball L et al. Bloodstream infections in critically ill patients with COVID-19. Eur J Clin Invest 2020: e13319. doi:10.1111/eci.13319

[5] Horby P, Landray M] et al.; RECOVERY Collaborative Group. Effect of Dexamethasone in Hospitalized Patients with COVID-19: Preliminary Report. medRxiv 22.06.2020. doi:10.1101/2020.06.22.20137273

[6] Lewis SR, Pritchard MW, Thomas CM et al. Pharmacological agents for adults with acute respiratory distress syndrome. Cochrane Database Syst Rev 2019; 7: CD004477

[7] Villar J, Ferrando C, Martínez D et al. Dexamethasone treatment for the acute respiratory distress syndrome: a multicentre, randomised controlled trial. Lancet Respir Med 2020; 8: 267-276. doi:10.1016/ S2213-2600(19)30417-5

[8] Papazian L, Forel J-M, Gacouin A et al. Neuromuscular blockers in early acute respiratory distress syndrome. N Engl J Med 2010; 363: 11071116

[9] Guérin C, Reignier J, Richard J-C et al. Prone positioning in severe acute respiratory distress syndrome. N Engl J Med 2013; 368: 21592168. doi:10.1056/NEJMoa1214103

[10] Combes A, Hajage D, Capellier G et al. Extracorporeal membrane oxygenation for severe acute respiratory distress syndrome. $N$ Engl J Med 2018; 378: 1965-1975. doi:10.1056/NEJMoa1800385

[11] Bornstein SR, Rubino F, Khunti K et al. Practical recommendations for the management of diabetes in patients with COVID-19. Lancet Diabetes Endocrinol 2020; 8: 546-550. doi:10.1016/S2213-8587(20) 30152-2

[12] Lloyd M, Karahalios A, Janus E et al. Improving Evidence-Based Treatment Gaps and Outcomes in Community-Acquired Pneumonia (IMPROVE-GAP) Implementation Team at Western Health. Effectiveness of a Bundled Intervention Including Adjunctive Corticosteroids on Outcomes of Hospitalized Patients with Community-Acquired Pneumonia: A Stepped-Wedge Randomized Clinical Trial. JAMA Intern Med 2019; 179: 1052-1060. doi:10.1001/jamainternmed.2019.1438 